\title{
Groups of flux-like transformations in loop quantum gravity
}

\author{
J. M. Velhinho \\ Departamento de Física, Universidade da Beira Interior \\ R. Marquês D’Ávila e Bolama, 6201-001 Covilhã, Portugal \\ jvelhi@ubi.pt
}

\begin{abstract}
We present a group of transformations in the quantum configuration space of loop quantum gravity that contains the set of all transformations generated by the flux variables.
\end{abstract}

\section{Introduction}

Besides the configuration variables, associated to holonomies, a key ingredient in loop quantum gravity (LQG) [1, 2] is the set of momentum variables, given by fluxes through appropriate surfaces. The flux variables correspond to 'infinitesimal translations' in the configuration space, and the one-parameter groups of transformations generated by each flux variable are well known [3, 4, 5]. However, the set of transformations obtained by exponentiation of the fluxes does not form a group.

Following our previous work [6, we present here a group of transformations that includes all of those generated by the flux variables. All transformations in this group leave the Ashtekar-Lewandowski measure invariant, and so they are all unitarily implemented in the standard LQG representation. In what follows, we will focus on the trivial bundle case and on the analytic set-up (see [6, 7, 8, for details).

\section{Preliminaries}

Let then $\Sigma$ be an analytic spatial manifold and $G$ a compact Lie group $(G=S U(2)$ in LQG). Consider oriented analytic curves in $\Sigma$, or more precisely, the set $\mathcal{E}$ of equivalence classes known as edges, where two curves are identified if they differ only by a (orientation preserving) reparametrization.

A generalized connection $\bar{A}$ is a map $\bar{A}: \mathcal{E} \rightarrow G$ such that $\bar{A}\left(e^{-1}\right)=(\bar{A}(e))^{-1}$ and $\bar{A}\left(e_{2} e_{1}\right)=\bar{A}\left(e_{2}\right) \bar{A}\left(e_{1}\right)$, whenever the composition of edges $e_{2} e_{1}$ is again an edge. (The composition of edges is induced by the natural composition of curves.) The set of all such maps $\bar{A}$ is the space of generalized connections $\overline{\mathcal{A}}$, which plays the role of a (kinematical) 'quantum configuration' space.

Let us introduce the notion of germ, for edges [2]. Consider the equivalence relation in $\mathcal{E}$ defined by: two edges are equivalent if they start at the same point and one of the edges is an analytic extension of the other. An equivalence class of edges is called a germ. 
So, a germ at a point $x$ is defined by the infinite set of Taylor coefficients, from which the whole family of equivalent edges can be reconstructed. The set of germs at any given point $x \in \Sigma$ is independent of $x$, and will be denoted by $\mathcal{K}$. The germ of $e$ is denoted by $[e]$.

\section{Basic group}

Since $G$ is a group, the set $\operatorname{Map}[\Sigma \times \mathcal{K}, G]$ of all maps $g: \Sigma \times \mathcal{K} \rightarrow G$ is a group under the pointwise product: $\left(g g^{\prime}\right)(x,[e]):=g(x,[e]) g^{\prime}(x,[e])$. We are interested in a subgroup thereof, defined as follows. For a given edge $e$ and a point $x$ on $e$, let $e_{x}$ denote the subedge of $e$ that starts at $x$. Now, for $g: \Sigma \times \mathcal{K} \rightarrow G$ and an edge $e$, let $S(g, e)$ denote the set of points $x$ along the edge $e$ such that $g\left(x,\left[e_{x}\right]\right)$ is different from the identity of the group $G$. The subgroup $\mathcal{F} \subset \operatorname{Map}[\Sigma \times \mathcal{K}, \mathrm{G}]$ is defined to be the set of all elements $g$ such that $S(g, e)$ is a finite set for every given edge e. It is clear that $\mathcal{F}$ is a subgroup, since if $S\left(g g^{\prime}, e\right)$ is an infinite set for some edge $e$, then either $S(g, e)$ or $S\left(g^{\prime}, e\right)$ (or both) must be infinite.

The following are examples of elements of the group $\mathcal{F}$.

Example 1: $g \in \operatorname{Map}[\Sigma \times \mathcal{K}, \mathrm{G}]$ supported on a finite number of points, i.e. such that $g$ is different from the identity of $G$ only in a finite number of points of $\Sigma$.

Example 2: Elements $g \in \operatorname{Map}[\Sigma \times \mathcal{K}, \mathrm{G}]$ supported in an analytic (or semianalityc) surface $S$, and such that $g$ also equals the identity for every germ that defines curves on the surface $S$. Appart from that, $g$ may vary from point to point on $S$ and depend on the germ at each point. (These elements are well defined, since the number of points $x$ in the intersection between an analytic edge $e$ and a (semi)analytic surface $S$ such that $\left[e_{x}\right]$ does not define curves in $S$ is finite.)

A subgroup $\mathcal{T F}$ of the group $\mathcal{F}$ is obtained when we consider functions $g \in \mathcal{F}$ that depend only on the tangent direction of the germ, i.e. such that, at each point, $g(x,[e])=$ $g\left(x,\left[e^{\prime}\right]\right)$ whenever germs $[e]$ and $\left[e^{\prime}\right]$ have the same tangent direction at the starting point $x$. The subgroup $\mathcal{T} \mathcal{F}$ can be defined exactly like $\mathcal{F}$, with $\mathcal{K}$ being replaced by the set $S^{2}$ of directions in the tangent space $T_{x} \Sigma$ at a point $x$.

Elements of $\mathcal{T} \mathcal{F}$ directly related to the LQG flux variables are defined as follows.

Example 3. Let $S$ be an oriented (semi)analytic surface and g a $G$-valued function on $S$. An element $g \in \mathcal{T F}$ is obtained by declaring that $g$ is supported on $S$ and, on points $x \in S:$ i) $g$ is the identity for every germ that defines curves on $S$; ii) $g(x,[e])=\mathrm{g}(x)$ for germs (that define curves) pointing upwards (with respect to the orientation of $S$ ); iii) $g(x,[e])=g(x)^{-1}$ for germs pointing downwards.

\section{Generalized flux transformations}

Our main result is that the group $\mathcal{F}$ can be seen as a group of transformations in the space $\overline{\mathcal{A}}$. In more precise terms, there is a faithful representation, hereby denoted $\Theta$, of $\mathcal{F}$ as a group of transformations in $\overline{\mathcal{A}}$. Moreover, the transformations generated by the LQG flux variables belong to the subgroup $\Theta(\mathcal{T} \mathcal{F})[\underline{6}$.

To define the representation $\Theta$, it is sufficient to give, for each $g \in \mathcal{F}$, the images $\Theta_{g}(\bar{A})$ of every $\bar{A} \in \overline{\mathcal{A}}$, which in turn are determined once $\Theta_{g}(\bar{A})(e)$ is known for every edge. In order to define $\Theta_{g}(\bar{A})(e)$, for any given $g, \bar{A}$ and edge $e$, one can use the same procedure as for the construction of graphs adapted to a given surface [2]. Essentially, given $g \in \mathcal{F}$ 
and an edge $e$, one works out a decomposition of the edge:

$$
e=e_{m}^{\epsilon_{m}} e_{m-1}^{\epsilon_{m-1}} \cdots e_{1}^{\epsilon_{1}}
$$

such that each subedge $e_{k}$ starts at a point of $S(g, e)$ and contains no other point of that set. Here, the symbols $\epsilon_{k}$ can take the values \pm 1 .

Once a decomposition of the type (1) is performed, it suffices to give the images of the edges $e_{k}$ in the decomposition:

$$
\Theta_{g}(\bar{A})\left(e_{k}\right)=\bar{A}\left(e_{k}\right) g^{-1}\left(x_{k},\left[e_{k}\right]\right), \quad k=1, \ldots, m,
$$

where $x_{k} \in S(g, e)$ is the starting point of the edge $e_{k}$. The images $\Theta_{g}(\bar{A})(e)$ are then determined by $\Theta_{g}(\bar{A})(e)=\left[\Theta_{g}(\bar{A})\left(e_{m}\right)\right]^{\epsilon_{m}}\left[\Theta_{g}(\bar{A})\left(e_{m-1}\right)\right]^{\epsilon_{m-1}} \cdots\left[\Theta_{g}(\bar{A})\left(e_{1}\right)\right]^{\epsilon_{1}}$.

It is straightforward to check that $\Theta$ is a representation, and so the group $\mathcal{F}$ can indeed be interpreted as a group of transformations in the space $\overline{\mathcal{A}}$. To see that $\mathcal{F}$ contains the transformations associated with the LQG fluxes, let us consider elements $g \in \mathcal{T F}$ of the type given in Example 3, with the $G$-valued functions $\mathrm{g}(x)$ being generated by $\operatorname{Lie}(G)$-valued functions $f(x)$, i.e. $g(x)=\exp (t f(x)), t \in \mathbb{R}$. The transformations $\Theta_{g}$ corresponding to these elements are precisely the transformations in $\overline{\mathcal{A}}$ generated by the LQG flux variables (see Eqs. (17) in [3]).

An important result is that, just like the transformations generated by the fluxes, all transformations in the group $\Theta(\mathcal{F})$ are continuous with respect to the natural topology of $\overline{\mathcal{A}}$, and leave the Ashtekar-Lewandowski measure invariant [6]. (The proof of this fact is essentially the same as in the case of the flux variables [3, 4, 9]). It follows that the standard LQG representation of the holonomy-flux algebra carries an unitary representation of the group $\Theta(\mathcal{F})$, which extends the set of unitary operators obtained by quantization of the exponentiated fluxes.

\section{References}

[1] A. Ashtekar and J. Lewandowski, Class. Quantum Grav. 21, R53 (2004).

[2] T. Thiemann, Modern Canonical Quantum General Relativity (Cambridge U. Press, Cambridge, 2007).

[3] J. Lewandowski, A. Okolow, H. Sahlmann and T. Thiemann, Commun. Math. Phys. 267, 703 (2006).

[4] C. Fleischhack, Commun. Math. Phys. 285, 67 (2009).

[5] B. Bahr and T. Thiemann, Class. Quantum Grav. 26, 235022 (2009).

[6] J. M. Velhinho, Int. J. Geom. Meth. Mod. Phys. 6, 479 (2009).

[7] J. M. Velhinho, Int. J. Geom. Meth. Mod. Phys. 1, 311 (2004).

[8] J. M. Velhinho, J. Geom. Phys. 41, 166 (2002).

[9] A. Okolow and J. Lewandowski, Class. Quantum Grav. 20, 3543 (2003). 